\title{
Spintronics with graphene-hexagonal boron nitride van der Waals heterostructures
}

\author{
M. Venkata Kamalakar, ${ }^{\text {a) }}$ André Dankert, Johan Bergsten, Tommy Ive, and Saroj P. Dash ${ }^{\text {b) }}$ \\ Department of Microtechnology and Nanoscience, Chalmers University of Technology, SE-41296 Göteborg, \\ Sweden
}

(Received 12 July 2014; accepted 13 November 2014; published online 25 November 2014)

\begin{abstract}
Hexagonal boron nitride (h-BN) is a large bandgap insulating isomorph of graphene, ideal for atomically thin tunnel barrier applications. In this letter, we demonstrate large area chemical vapor deposited (CVD) h-BN as a promising spin tunnel barrier in graphene spin transport devices. In such structures, the ferromagnetic tunnel contacts with h-BN barrier are found to show robust tunneling characteristics over a large scale with resistances in the favorable range for efficient spin injection into graphene. The non-local spin transport and precession experiments reveal spin lifetime $\approx 500 \mathrm{ps}$ and spin diffusion length $\approx 1.6 \mu \mathrm{m}$ in graphene with tunnel spin polarization $\approx 11 \%$ at $100 \mathrm{~K}$. The electrical and spin transport measurements at different injection bias current and gate voltages confirm tunnel spin injection through h-BN barrier. These results open up possibilities for implementation of large area CVD h-BN in spintronic technologies. (C) 2014 AIP Publishing LLC.
\end{abstract}

[http://dx.doi.org/10.1063/1.4902814]

With outstanding electronic transport properties, graphene has been anticipated to play a prominent role in the future nanoelectronic and spintronic applications. ${ }^{1}$ The prospect of graphene for spintronic devices emerges primarily from its inert nature to the spin of an electron. A low spinorbit coupling together with a negligible hyperfine interaction have led to predictions of extremely long spin relaxation length and spin lifetimes in graphene. ${ }^{2}$ Since the room temperature experimental demonstration of spin transport and precession in graphene, ${ }^{3}$ the field of graphene spintronics has witnessed several advancements. ${ }^{4-9}$ Enhancements in spin lifetimes of several hundred picosecond and diffusion lengths of more than $4 \mu \mathrm{m}$ have been reported using high mobility graphene device schemes. ${ }^{8,10}$ Similar enhancements have also been achieved through modification in a novel component of a spintronic device, called the tunnel barrier. The tunnel barrier is an ultrathin insulating layer, which when introduced between a ferromagnetic spin injector and graphene offers a solution to the abrupt conductivity mismatch at the interface, facilitating the spin injection to a great extent. ${ }^{11,12}$ Conventional oxide barriers such as $\mathrm{Al}_{2} \mathrm{O}_{3}, \mathrm{TiO}_{2}$, and $\mathrm{MgO}$ employed in majority of the studies ${ }^{3,5-7,13}$ so far have limitations related to pin holes and interface roughness, which contribute significantly to the spin relaxation. ${ }^{5,14,15}$

In the quest for improved interfaces, atomic layers of insulating two dimensional crystal based barriers such as hexagonal boron nitride (h-BN) have been proposed theoretically with novel predictions. ${ }^{16-19}$ Two dimensional (2D) h-BN is an ideal crystal for tunnel spin injection, because of a relatively large band gap $(\sim 6 \mathrm{eV})$ compared to other insulating layered crystals. ${ }^{20,21}$ Recent report by Yamaguchi et al. ${ }^{22}$ on spin injection into graphene using exfoliated h-BN revealed rather low spin lifetimes of 55 ps in graphene with tunnel spin polarization $\sim 1 \%-2 \%$, values similar to that observed

\footnotetext{
${ }^{\text {a)} E l e c t r o n i c ~ m a i l: ~ v e n k a t a . m u t t a @ c h a l m e r s . s e ~}$

${ }^{\mathrm{b})}$ Electronic mail: saroj.dash@chalmers.se
}

for transparent contacts. ${ }^{5}$ The observation of such low values was attributed to low resistance of the tunnel contacts in their experiments, which do not address the conductivity mismatch appropriately. Thus efficient spin injection along with the possibility of observation of high spin lifetimes using 2D crystals h-BN still remains an experimental challenge. In this letter, we report an order of magnitude enhancement in spin lifetime in graphene and tunnel spin polarization using large area chemical vapor deposited (CVD) h-BN.

To demonstrate the viability of CVD h-BN as a tunnel barrier for spin injection into graphene, we fabricated lateral spin transport devices of graphene with ferromagnetic Co electrodes having a CVD h-BN interface layer. The scheme of a non-local ${ }^{3}$ graphene/h-BN van der Waals heterostructure spin transport device is presented in Fig. 1(a). We employed CVD h-BN (procured from graphene supermarket) on copper substrate with large coverage area. The CVD h-BN on copper foil was first spin coated with a thick layer of polymethyl methacrylate (PMMA) resist. The copper layer was then etched away in a solution of $\mathrm{FeCl}_{3}$, followed by cleaning in $10 \% \mathrm{HCl}$ and deionized water. The h-BN layer supported by the PMMA resist was then transferred onto graphene flakes exfoliated on $\mathrm{SiO}_{2} / \mathrm{Si}$ chip. Post transfer and drying, the chip was baked at $150^{\circ} \mathrm{C}$ for $10 \mathrm{~min}$ to improve the adhesion of the transferred film. The chip was finally cleaned with acetone to remove the PMMA resist layer and further annealed at $400{ }^{\circ} \mathrm{C}$ in $\mathrm{Ar} / \mathrm{H}_{2}$ atmosphere to remove any possible polymer residues. A first step of ebeam lithography was performed to pattern h-BN to regions covering graphene flakes. The ferromagnetic $\mathrm{Co} / \mathrm{Au}(65 \mathrm{~nm} /$ $15 \mathrm{~nm}$ ) electrodes were then fabricated by a 2nd step of ebeam lithography, followed by metal evaporation and liftoff. In Fig. 1(b), we present an optical microscope image of a fabricated device clearly showing a patterned layer of h$\mathrm{BN}$ covering a graphene flake with top $\mathrm{Co} / \mathrm{Au}$ contacts. A schematic representation of the device fabrication process is displayed in Fig. 1(c). 
(a)

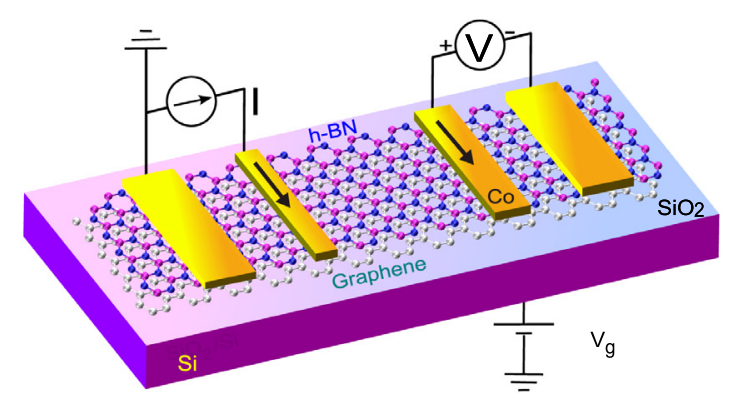

(b)

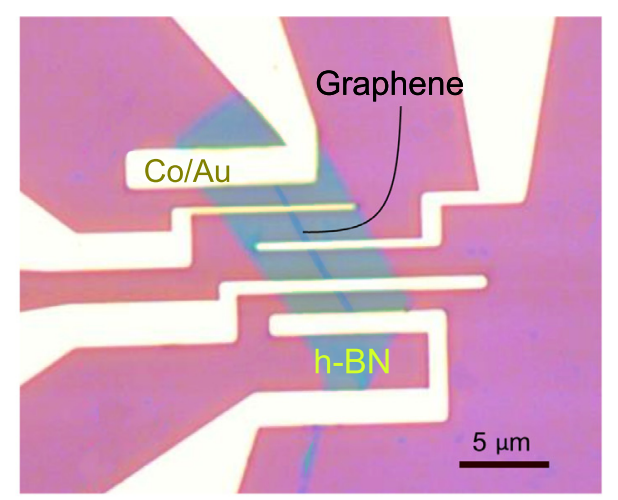

(c)

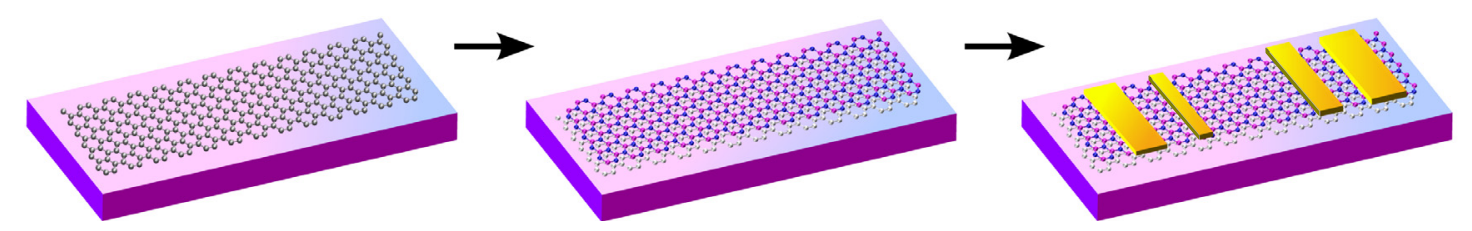

FIG. 1. Spintronic Device with graphene-h-BN van der Waals heterostructures. (a) Spintronic device scheme showing graphene and h-BN heterostructures with ferromagnetic contacts in non-local measurement geometry. (b) Optical microscope image of a fabricated graphene/h-BN/Co device. (c) Schematic representation of the main steps in device fabrication.

Prior to spin transport experiments, we electrically assessed the quality of our devices in different measurement configurations. Efficient spin injection requires the tunnel barrier resistance to be in the optimum range as governed by the conductivity mismatch issue. ${ }^{11,12}$ The tunnel resistance in our devices was measured by three-terminal (3T) measurement geometry at different temperatures as shown in Fig. 2(a). The measured current-voltage (I-V) characteristics are typical for tunnel barriers with weak temperature dependence having contact resistance $\mathrm{R}_{\mathrm{C}} \sim 10 \mathrm{k} \Omega \mu \mathrm{m}^{2}$. The gate dependence of the tunnel contact resistance (shown in the lower inset of Fig. 2(a)) indicates a fairly stable resistance over a large range of gate sweep voltage. In Fig. 2(b), we display the atomic force microscopy image of a region showing effective thickness of the CVD h-BN layer on $\mathrm{SiO}_{2} / \mathrm{Si}$ substrate. Some variation in the contact resistances was noticed, which can originate from the non-uniformity in thickness of h-BN layer. ${ }^{20}$ In spite of local variations in thickness, the (a)

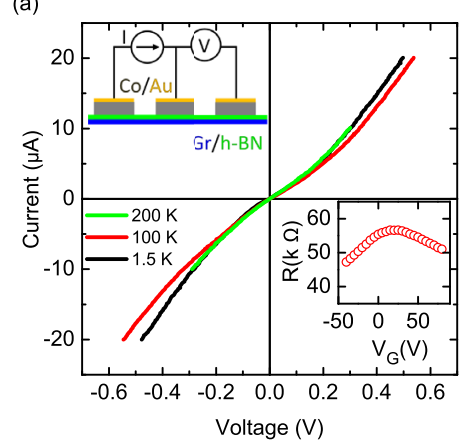

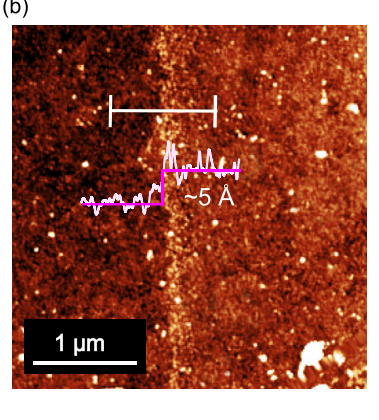

FIG. 2. Characterization of h-BN tunnel barrier. (a) Current-Voltage characteristic curves of an h-BN/Co tunnel contact on graphene at different temperatures. Upper inset: 3T measurement geometry, Lower inset: Gate voltage dependence of the tunnel contact resistance. (b) Atomic force microscopy image showing effective thickness of $\sim 5 \AA$ of the transferred h$\mathrm{BN}$ layer on $\mathrm{SiO}_{2}$ substrate. values of tunnel resistance found in our devices are typically higher than graphene channel spin resistance ${ }^{23}$ and are in the optimum range (as estimated from the Fert-Jaffrès mode ${ }^{11}$ ) to circumvent the conductivity mismatch problem. ${ }^{11,12}$ The graphene channel resistance of $\sim 4.5 \mathrm{k} \Omega$ was measured in four-probe configuration, which showed regular gate modulation yielding a carrier mobility $\sim 2000 \mathrm{~cm}^{2} \cdot \mathrm{V}^{-1} \cdot \mathrm{s}^{-1}$.

In order to demonstrate tunnel spin injection through CVD h-BN barrier, we performed non-local (NL) spin valve and Hanle spin precession measurements. ${ }^{3}$ The NL measurements ensure pure signal measurement devoid of other possible magneto-resistive signatures. In Fig. 3(a), we show a typical spin valve signal obtained on a device by sweeping an in-plane magnetic field along the length of the electrodes. We obtained clear switching in the spin signal yielding a NL resistance change $\Delta R_{N L}=\Delta V_{N L} / I$ of $5 \Omega$ between the parallel and anti-parallel configurations of the ferromagnetic injector and detector electrodes. The small asymmetry in switching fields observed between forward and backward sweep could be attributed to possible asymmetry/exchange bias in the injector and detector electrodes.

The value of spin signal is significantly higher than previous report using exfoliated h-BN barrier. ${ }^{22}$ Next, we performed Hanle spin precession measurement to evaluate the spin lifetime $\tau_{\mathrm{s}}$ and the diffusion length $\lambda$ in graphene. Here, we swept an out of plane magnetic field to achieve precession of the spin diffusing from injector to detector electrodes. ${ }^{3}$ Fig. 3(b) displays the modulation of spin signal due to the Hanle spin precession in the $2 \mu \mathrm{m}$ long graphene channel at $100 \mathrm{~K}$. In order to evaluate the spin lifetime and the diffusion constant, we analyzed the data using the analytical expression (Eq. (1)) of spin precession encompassing the diffusion and the relaxation terms. ${ }^{3,5}$

$$
R_{N L} \propto \int_{0}^{\infty} \frac{1}{\sqrt{4 \pi D t}} e^{-\frac{L^{2}}{4 D t}} \cos \left(\omega_{L} t\right) e^{-\left(\frac{t}{\tau_{s}}\right)} d t
$$


(a)

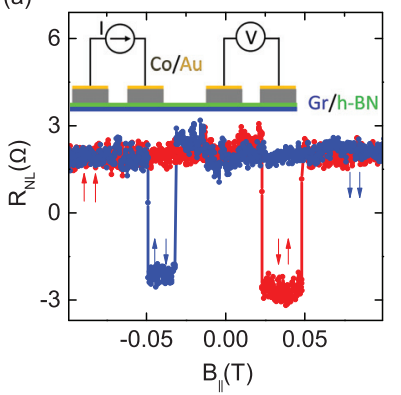

(b)

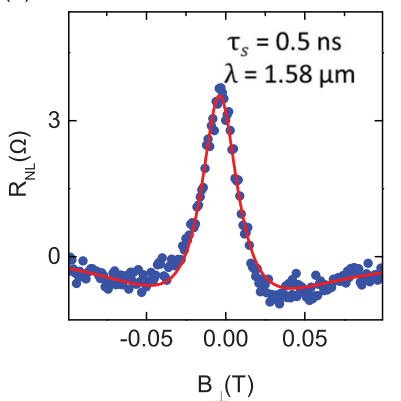

(c)
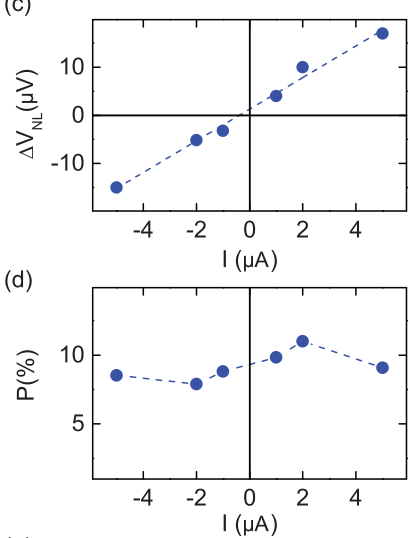

(e)

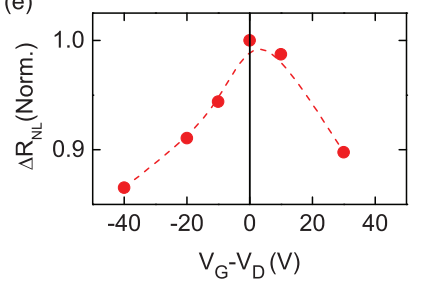

FIG. 3. Tunnel spin injection and transport in $\mathrm{Co} / \mathrm{hBN} / \mathrm{Graphene}$ devices. (a) Non-local spin valve resistance recorded with in-plane magnetic field along the length of the electrodes, (b) Hanle spin precession signal measured as a function of out of plane magnetic field at $100 \mathrm{~K}$, (c) spin valve signal as a function of applied injection current bias. (d) Spin polarization as a function of injection current. (e) Normalized spin signal as a function of applied gate voltage $\mathrm{V}_{\mathrm{G}}-\mathrm{V}_{\mathrm{D}}\left(\mathrm{V}_{\mathrm{D}}\right.$ being the Dirac point $)$.

where $\omega_{L}=\frac{g \mu_{B}}{\hbar} B_{\perp}$ (with Lande's g-factor $\mathrm{g}=2$ ) is the Larmor frequency of spin precession in a perpendicular magnetic field $B_{\perp}$. With an effective length of the graphene channel $L=2 \mu \mathrm{m}$, we obtained a spin lifetime $\tau_{s} \approx 500 \mathrm{ps}$ and a diffusion constant $D=0.005 \mathrm{~m}^{2} \mathrm{~s}^{-1}$ yielding a spin diffusion length $\lambda=\sqrt{D \tau_{s}}$ of $1.58 \mu \mathrm{m}$. The observed spin lifetime is nearly ten times higher than the previous report of exfoliated $\mathrm{h}$-BN layers ${ }^{22}$ and is comparable to some of the best spin lifetimes observed using conventional oxide tunnel barriers. ${ }^{4,8,14}$

Spin valve measurements performed with several injection bias currents show reasonably linear enhancement of spin signal as shown in Fig. 3(c). To estimate the spin polarization of our contacts, we utilized the exponential decay ${ }^{3}$ of the spin signal in a channel with conductivity $\sigma$ and width $W$, described by

$$
\Delta R_{N L}=\frac{P^{2} \lambda}{2 W \sigma} \exp (-L / \lambda)
$$

We obtained spin polarization $\mathrm{P}$ up to $11 \%$ for $2 \mu \mathrm{A}$ of injection current (Fig. 3(d)). Note that except for a few occasional reports of high spin polarization created in graphene $\left(\mathrm{P} \sim 30 \%\right.$ with $\mathrm{MgO}$ based barriers $\left.{ }^{4}\right)$, the routine values lie mostly below $15 \%{ }^{24,25}$ The value obtained using our $2 \mathrm{D}$ CVD h-BN barrier is an order higher in magnitude than what has been obtained on transparent contacts and earlier exfoliated h-BN reports. ${ }^{22} \mathrm{~A}$ systematic investigation showing the enhancement of spin signals from transparent contacts to high resistance h-BN is presented elsewhere. ${ }^{26}$ At low bias voltages $(< \pm 120 \mathrm{mV})$, we observed a change of $10 \%-20 \%$ in polarization on either side of the maxima. Such change in spin polarization is similar to the values generally observed

in $\mathrm{MgO}$ and $\mathrm{Al}_{2} \mathrm{O}_{3}$ based magnetic tunnel junction in low bias regime. ${ }^{27,28}$ In addition, as shown in Fig. 3(e), we also observe a variation in the spin signal with gate voltage, a decrease of spin signal $\approx 10 \%$ at higher gate voltages. Such behavior is typical for spin tunnel barriers with resistance much higher than graphene channel resistance. ${ }^{4}$

The spin parameters obtained in our device indicate that an improved performance can be achieved employing CVD h-BN tunnel barriers. The fabrication process also does not involve unintentional doping of graphene near the contacts, an issue that remains unaddressed with conventional metal oxide barrier deposition. In comparison to graphene based barriers ${ }^{29-33}$ h-BN has relatively higher bandgap, which underscores its importance in 2D crystal based future spintronic devices. Such tunnel barriers together with schemes of improving graphene channel transport properties ${ }^{8,10}$ and fine tuning of the tunnel resistance can potentially lead to further enhancements in the performance of spintronic devices. In addition, our work demonstrates that tunnel interfaces suitable for spin injection at a large scale can be achieved using CVD grown h-BN with a high technological relevance. The current demonstration encourages studies on spin injection into other important materials such as semiconducting $2 \mathrm{D}$ crystals $^{34}$ and silicon. ${ }^{35}$

In conclusion, we have fabricated non-local graphene spin transport devices using chemical vapor deposited hexagonal boron nitride tunnel contacts. Reproducible and robust non-linear tunneling characteristics with weak temperature dependence of the tunnel resistance have been observed over a chip scale. The resistances of the tunnel contacts are found to be in the comfortable range for spin injection into graphene, bypassing the conductivity mismatch hurdle. With tunnel contacts $\approx 10 \mathrm{k} \Omega$, we observed a non-local resistance of $5 \Omega$, spin lifetime $\tau \approx 500 \mathrm{ps}$, spin diffusion length $\lambda \approx 1.6 \mu \mathrm{m}$ and a polarization $\approx 11 \%$ at $100 \mathrm{~K}$. The spin transport measurements at different injection bias current and gate voltages confirms tunneling nature of spin injection through h-BN barriers. These results are extremely promising for a CVD grown 2D material considering its advantage for large scale fabrication. Our research further opens ways for investigation of spin filtering attributes of h-BN tunnel barriers and its implementation in spintronic devices.

The authors acknowledge the support from colleagues of Quantum Device Physics Laboratory and Nanofabrication Laboratory at Chalmers University of Technology. This research was financially supported by Nano Area of Advance program at Chalmers University of technology, EU FP7 Marie Curie Career Integration grant, and Swedish Research Council Young Researcher Grant.

${ }^{1}$ K. S. Novoselov, V. I. Fal'ko, L. Colombo, P. R. Gellert, M. G. Schwab, and K. Kim, Nature 490, 192 (2012).

${ }^{2}$ D. Huertas-Hernando, F. Guinea, and A. Brataas, Phys. Rev. B 74, 155426 (2006).

${ }^{3}$ N. Tombros, C. Jozsa, M. Popinciuc, H. T. Jonkman, and B. J. van Wees, Nature 448, 571 (2007).

${ }^{4}$ W. Han, K. Pi, K. M. McCreary, Y. Li, J. J. I. Wong, a. G. Swartz, and R. K. Kawakami, Phys. Rev. Lett. 105, 167202 (2010).

${ }^{5}$ W. Han, K. M. McCreary, K. Pi, W. H. Wang, Y. Li, H. Wen, J. R. Chen, and R. K. Kawakami, J. Magn. Magn. Mater. 324, 369 (2012). 
${ }^{6}$ A. Avsar, T.-Y. Yang, S. Bae, J. Balakrishnan, F. Volmer, M. Jaiswal, Z. Yi, S. R. Ali, G. Güntherodt, B. H. Hong, B. Beschoten, and B. Özyilmaz, Nano Lett. 11, 2363 (2011).

${ }^{7}$ T. Maassen, J. J. van den Berg, N. Ijbema, F. Fromm, T. Seyller, R. Yakimova, and B. J. van Wees, Nano Lett. 12, 1498 (2012).

${ }^{8}$ P. J. Zomer, M. H. D. Guimarães, N. Tombros, and B. J. van Wees, Phys. Rev. B 86, 161416 (2012).

${ }^{9}$ I. Neumann, J. Van de Vondel, G. Bridoux, M. V. Costache, F. Alzina, C. M. Sotomayor Torres, and S. O. Valenzuela, Small 9, 156 (2013).

${ }^{10}$ M. H. D. Guimarães, A. Veligura, P. J. Zomer, T. Maassen, I. J. VeraMarun, N. Tombros, and B. J. van Wees, Nano Lett. 12, 3512 (2012).

${ }^{11}$ A. Fert and H. Jaffrès, Phys. Rev. B 64, 184420 (2001).

${ }^{12}$ G. Schmidt, D. Ferrand, L. Molenkamp, A. Filip, and B. van Wees, Phys. Rev. B 62, R4790 (2000).

${ }^{13}$ A. Dankert, M. V. Kamalakar, J. Bergsten, and S. P. Dash, Appl. Phys. Lett. 104, 192403 (2014).

${ }^{14}$ W. Han and R. K. Kawakami, Phys. Rev. Lett. 107, 047207 (2011).

${ }^{15}$ S. P. Dash, S. Sharma, J. C. Le Breton, J. Peiro, H. Jaffrès, J.-M. George, A. Lemaitre, and R. Jansen, Phys. Rev. B 84, 054410 (2011).

${ }^{16}$ V. M. Karpan, G. Giovannetti, P. Khomyakov, M. Talanana, A. A. Starikov, M. Zwierzycki, J. van den Brink, G. Brocks, and P. J. Kelly, Phys. Rev. Lett. 99, 176602 (2007).

${ }^{17}$ V. M. Karpan, P. A. Khomyakov, G. Giovannetti, A. A. Starikov, and P. J. Kelly, Phys. Rev. B 84, 153406 (2011).

${ }^{18}$ M. L. Hu, Z. Yu, K. W. Zhang, L. Z. Sun, and J. X. Zhong, J. Phys. Chem. C 115, 8260 (2011).

${ }^{19}$ O. Yazyev and A. Pasquarello, Phys. Rev. B 80, 035408 (2009).

${ }^{20}$ L. Britnell, R. V. Gorbachev, R. Jalil, B. D. Belle, F. Schedin, M. I. Katsnelson, L. Eaves, S. V. Morozov, A. S. Mayorov, N. M. R. Peres, A. H. C. Neto, J. Leist, A. K. Geim, L. A. Ponomarenko, and K. S. Novoselov, Nano Lett. 12, 1707 (2012).
${ }^{21}$ A. K. Geim and I. V. Grigorieva, Nature 499, 419 (2013).

${ }^{22}$ T. Yamaguchi, Y. Inoue, S. Masubuchi, S. Morikawa, M. Onuki, K. Watanabe, T. Taniguchi, R. Moriya, and T. Machida, Appl. Phys. Express 6, 073001 (2013).

${ }^{23}$ B. Dlubak, M.-B. Martin, C. Deranlot, B. Servet, S. Xavier, R. Mattana, M. Sprinkle, C. Berger, W. a. De Heer, F. Petroff, A. Anane, P. Seneor, and A. Fert, Nat. Phys. 8, 557 (2012).

${ }^{24}$ F. Volmer, M. Drögeler, E. Maynicke, N. von den Driesch, M. L. Boschen, G. Güntherodt, and B. Beschoten, Phys. Rev. B 88, 161405 (2013).

${ }^{25}$ Y. P. Liu, H. Idzuchi, Y. Fukuma, O. Rousseau, Y. Otani, and W. S. Lew, Appl. Phys. Lett. 102, 033105 (2013).

${ }^{26}$ M. V. Kamalakar, A. Dankert, J. Bergsten, T. Ive, S. P. Dash, and M. Venkata Kamalakar, Sci. Rep. 4, 6146 (2014).

${ }^{27}$ S. Yuasa, T. Nagahama, A. Fukushima, Y. Suzuki, and K. Ando, Nat. Mater. 3, 868 (2004).

${ }^{28}$ J. Moodera, J. Nowak, and R. van de Veerdonk, Phys. Rev. Lett. 80, 2941 (1998).

${ }^{29}$ E. Cobas, A. L. Friedman, O. M. J. Van't Erve, J. T. Robinson, and B. T. Jonker, Nano Lett. 12, 3000 (2012).

${ }^{30}$ O. M. J. van't Erve, A. L. Friedman, E. Cobas, C. H. Li, J. T. Robinson, and B. T. Jonker, Nat. Nanotechnol. 7, 737 (2012).

${ }^{31}$ A. L. Friedman, O. M. J. van't Erve, C. H. Li, J. T. Robinson, and B. T. Jonker, Nat. Commun. 5, 3161 (2014).

${ }^{32}$ B. Dlubak, M.-B. Martin, R. S. Weatherup, H. Yang, C. Deranlot, R. Blume, R. Schloegl, A. Fert, A. Anane, S. Hofmann, P. Seneor, and J. Robertson, ACS Nano 6, 10930 (2012).

${ }^{33}$ F. Godel, M. Venkata Kamalakar, B. Doudin, Y. Henry, D. Halley, and J.F. Dayen, Appl. Phys. Lett. 105, 152407 (2014).

${ }^{34}$ A. Dankert, L. Langouche, M. V. Kamalakar, and S. P. Dash, ACS Nano 8, 476 (2014).

${ }^{35}$ A. Dankert, R. S. Dulal, and S. P. Dash, Sci. Rep. 3, 3196 (2013). 